\title{
MFGE8 regulates TGF- $\beta$-induced epithelial mesenchymal transition in endometrial epithelial cells in vitro
}

\author{
Liang $\mathrm{Yu}^{1,5}$, Rong $\mathrm{Hu}^{2}$, Claretta Sullivan ${ }^{3}, \mathrm{R}$ James Swanson ${ }^{4}$, Sergio Oehninger ${ }^{1}$, \\ Ying-Pu Sun ${ }^{5}$ and Silvina Bocca ${ }^{1}$
}

${ }^{1}$ The Jones Institute for Reproductive Medicine, Department of Obstetrics and Gynecology, Eastern Virginia Medical School, Norfolk, Virginia, USA, ${ }^{2}$ Reproductive Medicine Center, Key Laboratory of Fertility Preservation and Maintenance of Ministry of Education, Ningxia Medical University, General Hospital of Ningxia Medical University, Yinchuan, China, ${ }^{3}$ Department of Surgery, Eastern Virginia Medical School, Norfolk, Virginia, USA, ${ }^{4}$ Department of Biological Sciences, Old Dominion University, Norfolk, Virginia, USA, and ${ }^{5}$ Reproductive Medical Center, The First Affiliated Hospital of Zhengzhou University, Zhengzhou, China

Correspondence should be addressed to S Bocca or to Y-P Sun; Emails: boccas@evms.edu or syp2008@vip.sina.com

\begin{abstract}
This study investigated the role of milk fat globule-epidermal growth factor-factor 8 (MFGE8) in TGF- $\beta$-induced epithelialmesenchymal transition (EMT) of endometrial epithelial cells. These were in vitro studies using human endometrial epithelial cells and mouse blastocysts. We investigated the ability of TGF- $\beta$ to induce EMT in endometrial epithelial cells (HEC-1A) by assessment of cytological phenotype (by light and atomic force microscopy), changes in expression of the markers of cell adhesion/differentiation E- and N-cadherin, and of the transcription factor Snail (by immunofluorescence and immunoblotting), and competence to support embryo attachment in a mouse blastocyst outgrowth assay. We also studied the effects of E-cadherin expression in cells transfected by retroviral shRNA vectors specifically silencing MFGE8. Results demonstrated that TGF- $\beta$ induced EMT as demonstrated by phenotypic cell changes, by a switch of cadherin expression as well as by upregulation of the expression of the mesenchymal markers Snail and Vimentin. Upon MFGE8 knockdown, these processes were interfered with, suggesting that MFGE8 and TGF- $\beta$ together may participate in regulation of EMT. This study demonstrated for the first time that endometrial MFGE8 modulates TGF- $\beta$-induced EMT in human endometrium cells.
\end{abstract}

Reproduction (2016) 152 225-233

\section{Introduction}

Milk fat globule-epidermal growth factor-factor 8 (MFGE8), originally found in milk and mammary epithelial cells (Stubbs et al. 1990), is also known as lactadherin (Taylor et al. 1997) or SED1 (Ensslin \& Shur 2003). Our group was the first to report the expression of MFGE8 in human endometrium and its upregulation during the window of implantation (Mirkin et al. 2005). Our data showed that MFGE8 protein is upregulated by prolactin in primary endometrial epithelial cell cultures, indicating paracrine epithelial-stromal cells interaction (Franchi et al. 2011). In addition, MFGE8 protein is highly expressed in human chorionic villi at all trimesters of gestation and in murine implantation sites (Bocca et al. 2012), and participates in trophoblast adhesion with its receptor integrin $\alpha \nu \beta 3$ in an in vitro model of human implantation (Schmitz et al. 2014). We have also demonstrated that endometrial MFGE8 gene expression, as well as the expression of other inflammatory factors, such as IL-6 and IL-8, are significantly regulated by TNF $\alpha$ (Yu et al. 2014) and hCG (Sarhan A et al. 2013) respectively.

These studies strongly suggested that endometrial MFGE8 performs an important role in physiological conditions during menstrual endometrium remodeling and implantation, and dysfunctions of its expression may be associated with endometrial pathological conditions. In extra-uterine tissues, this secreted phosphatidylserine binding protein, MFGE8, has been reported to have functions in apoptosis control, cell remodeling, neovascularization and immunomodulation (Morris et al. 1983, Silvestre et al. 2005). In human melanoma cells, MFGE8 promoted disease progression through coordination with integrin $\alpha \nu \beta 3$ in tumor microenvironment; it also increased cell resistance to apoptosis, triggered epithelialmesenchymal transition (EMT), and stimulated invasion (Jinushi et al. 2008). However, melanoma cells with MFGE8 deficiency showed the ability to attenuate Akt 
and Twist signaling and thereby compromise tumor cell survival, EMT and invasion.

Epithelial-mesenchymal transition (EMT) plays a fundamental role in embryonic development and in tissue repair. Several lines of evidence also suggest that EMT is necessary for the emergence of cancer stem cells and for tumor progression (Thiery et al. 2009). EMT is characterized by a loss of the apical-basal polarity of epithelial cells, remodeling of the cytoskeleton, the emergence of a mesenchymal phenotype and increased cell mobility. The proteins that form tight junctions and adherent junctions such as occludin, claudin and E-cadherin disappear during EMT, and mesenchymal markers begin to be expressed. Features of EMT in epithelial cells include alteration of cytoskeleton structure and morphology by losing cortical actin, developing large lamellipodia and increasing their spreading on the underlying substratum (Radisky et al. 2005).

EMT can be initiated in response to a variety of signals, such as transforming growth factor- $\beta$ (TGF- $\beta$ ), insulin growth factor 2 (IGF2) or epidermal growth factor (EGF). These EMT inducers lead to the activation of transcription factors, including Snail and Slug, ZEB1/2, and Twist (Bertrand et al. 2015). Specific transcription factors, which repress the transcription of E-cadherin, have been identified. The zinc-finger protein Snail1 and its close relative Slug (Snail2) were some of the first transcription factors to be identified. Twist, a member of the basic helix-loop-helix (bHLH) transcription factor family, has long been known to trigger EMT, and Twist seems to be necessary for the metastasis of E-cadherindeficient cells (Bartley et al. 2014).

Human embryo implantation is a critical multistep process consisting of embryo apposition/adhesion, followed by trophoblast penetration and invasion. In a recently published study using an in vitro model of attachment between Ishikawa cells and Jar spheroids, sexual ovarian steroid hormones (17 $\beta$-estradiol and progesterone) resulted in upregulation of $\mathrm{N}$-cadherin and vimentin, a mesenchymal cell marker, with concomitant downregulation of E-cadherin in the Ishikawa cells, accelerated Ishikawa cells motility, increased JAR spheroid outgrowth, and redistribution of $\mathrm{N}$-cadherin effects which were most prominent in proximity to the adhered spheroids. This strongly suggested activation of EMT (Uchida et al. 2012). As such, these authors concluded that their results collectively suggested that the ovarian sex steroids and the implanting embryo (Jar spheroids mimicking trophoblast) induced EMT of endometrial epithelial cells, and that these processes may play a pivotal role in early human embryo implantation via functional control of $\mathrm{N}$-cadherin.

TGF- $\beta$ superfamily proteins have been demonstrated to play a crucial role in inducing EMT and in almost all major developmental processes depending on EMT (Yang \& Weinberg 2008), such as embryo implantation, embryogenesis and organ development. TGF- $\beta$ is preferentially synthesized in the stroma and differentially expressed in the human endometrium regulated by the sex hormones estrogen and progesterone (Jones et al. 2006, Omwandho et al. 2010). These growth factors are also abundantly detected by epithelial glands and trophoblast cells secreted into the uterine fluid, where they are involved in epithelium remodeling to establish a successful pregnancy (Chea et al. 2005). Researchers found that cellular responses to TGF- $\beta$ stimulation are determined by the presence of TGF- $\beta$ type I and type II receptor complexes; therefore, unresponsiveness to TGF- $\beta$ may be caused by mutational inactivation or lack of receptors that are characterized in many human cancers (Massague et al. 2000, Piestrzeniewicz-Ulanska et al. 2002). Although TGF- $\beta$ is shown to modulate the invasive properties of tumor cells (Van Themsche et al. 2007), little information is available regarding the effect of TGF- $\beta$ on the invasiveness and receptivity of endometrial epithelial cells.

In this study, we investigated the in vitro role of MFGE8 in TGF- $\beta$ induced EMT in endometrial epithelial cells, hypothesizing that MFGE8 would regulate EMT and potentially promote embryo attachment and early invasion. We first tested the ability of TGF- $\beta$ to induce EMT in endometrial epithelial cells by studying cytological phenotypes, changes in markers of cell adhesion/differentiation, and the ability to support embryo attachment. These data demonstrate that TGF- $\beta$ induces EMT junction changes and promotes embryo attachment. Second, when MFGE8 was knocked down in endometrial epithelial cells, the EMT process and the interaction between embryo and human endometrial epithelial cells as triggered by TGF- $\beta$ were interfered, supporting the concept that MFGE8 may play a modularity role during trophoblast attachment and early invasion, and endometrial remodeling.

\section{Materials and methods}

\section{Endometrial epithelial cell culture}

The human endometrial epithelial adenocarcinoma HEC-1A (ATCC HTB112) has been characterized as an in vitro model for EMT and trophoblast adhesion as described previously (Tsukamoto et al. 2007, Paule et al. 2012). HEC-1A cells were cultured in McCoy's medium with 10\% fetal bovine serum in a humidified atmosphere of $5 \% \mathrm{CO}_{2}$ incubator at $37^{\circ} \mathrm{C}$. To detect the effects of TGF- $\beta$ on EMT, HEC-1A cells were cultured in the presence of $20 \mathrm{ng} / \mathrm{mL}$ TGF- $\beta$ (Invitrogen, PHG9204) (Rhyu et al. 2005, Cheng et al. 2012). The studies were considered exempt from Institutional Review Board approval because established cell lines were used.

\section{Immunofluorescence}

HEC-1A was characterized as an endometrial epithelial cell line by immunofluorescence (IF), staining with antibodies including ER- $\beta$ (Abcam, ab3576), Prl-R (Abcam, ab170935), hCG-R 
(Abcam, ab96603), MFGE8 (Abcam, ab17787), cytokeratin (Abcam, ab59400) and vimentin (Santa Cruz, sc6260) as markers for epithelial and mesenchymal cells respectively (all 1:200) (Supplementary Material and Supplementary Fig. 1, see section on supplementary data given at the end of this article).

To evaluate the effects of TGF- $\beta$ on EMT in HEC-1A cells, antibodies used for immunofluorescence were primary anti-E and anti-N-cadherin antibodies (1:200; BD Biosciences, San Jose, CA, USA, E-cadherin 610181, N-cadherin 610920). HEC-1A cells were cultured in 8 well chamber slides before fixation in $4 \%$ paraformaldehyde. Immunofluorescence was performed as described previously (Yu et al. 2014). Briefly, nonspecific binding sites were blocked in PBS containing 5\% bovine serum albumin. Primary antibodies were added in blocking buffer for overnight at $4{ }^{\circ} \mathrm{C}$. After washing, fluorescein isothiocyanate-conjugated secondary antibody (1:400; Santa Cruz Biotechnology) was added for 1 -h incubation at room temperature. Coverslips were mounted onto glass slides using mounting medium containing DAPI and were visualized using a fluorescence microscope, Olympus BX50 with a DP-70 Olympus camera. A nonimmune IgG control antibody (normal mouse ascites; clone NS-1, Sigma) was used as negative control.

\section{Atomic force microscopy analysis}

HEC-1A cell morphology was examined by light microscopy and by atomic force microscopy (AFM). High-resolution AFM analysis was used to characterize cell surface with its contact probe mechanism. AFM images were collected with an Agilent 5500 scanning probe microscope (Agilent Technologies) as described previously (Sarhan A et al. 2013). Silicon nitride cantilevers $(0.01-0.1 \mathrm{~N} / \mathrm{m})$ were used to scan HEC-1A in contact mode at a rate of 0.5-2.0 lines per second at 512 points per line resolution. Images were processed as first-order flattened. Changes in cell surface topography were quantified using a cell height transect line across similar regions of the HEC-1A. The topographical and morphological change on HEC-1A cell was analyzed to reveal the effects of TGF- $\beta$ treatment.

\section{Embryo outgrowth in an in vitro attachment/invasion assay}

Because of the current ethical dilemma of using human embryos for research, we performed an in vitro attachment/ invasion assay with mouse embryos. Four to eight weeks old CBA/B6 F1 females mice were superovulated by intraperitoneal injection of $5 \mathrm{IU}$ of pregnant mare's serum gonadotropin (PMSG) followed $48 \mathrm{~h}$ later by $5 \mathrm{IU}$ of human chorionic gonadotropin (hCG). Females were paired with CD1 males of proven fertility, and pregnancy was confirmed by the presence of a vaginal copulation plug 12-h post-hCG injection, then 2 cell embryos were collected 34-h post-hCG injection and cultured in Krebs medium to the blastocyst stage at $37^{\circ} \mathrm{C}, 5 \%$ $\mathrm{CO}_{2}, 100 \% \mathrm{H}_{2} \mathrm{O}$, in 96 well plates overlaid by toxicity-tested heavy paraffin oil. Experiments were conducted in accordance with the NIH standards and with approval from appropriate Animal Care Committees (IACUC \#11-005).
Mouse embryos at the blastocyst stage were placed in co-culture with a confluent HEC-1A monolayer with fresh medium, and were monitored up to $72 \mathrm{~h}$ as described previously (Uchida et al. 2012, Burch et al. 2014, Green et al. 2015). At 0-, 24- and 72-h culture, the blastocysts' hatching and outgrowth were monitored under an inverted phase contrast microscope (Olympus Optical) and the adhesion was detected at $48 \mathrm{~h}$. At the end of culture, for the adhered and spread blastocysts, the relative outgrowth area was measured using Image J as described previously (Qin et al. 2005, Kawamura et al. 2012). The area per blastocyst outgrowth was calculated from the treated group then relative to the control group, and compared for statistical analysis.

\section{shRNA transfection in HEC-1A cells}

Knockdown of MFGE8 in HEC-1A cells was performed by transfection of a set of shRNA constructs containing 4 vials of gene-specific shRNA expression vectors in retroviral silencing plasmid (OriGene, Rockville, MD, USA) respectively. A negative control with non-effective shRNA cassette was used as a control. HEC-1A cells were transfected with the vectors using TurboFectin 8.0 transfection reagent (OriGene, Rockville, MD, USA). The designed MFGE8 shRNA sequences are

\section{M1: CCTGTGGAGGCTCAGTACGTGAGATTGTA; M2: GGCTCTGTCCAGTTTGTGGCATCCTACAA; M3: TGGCCTACAGCCTTAATGGACACGAATTC; M4: GGCAACCACTGTGAGACGAAATGTGTCGA.}

HEC- 1 A cells $\left(2 \times 10^{5}\right.$ cells/well $)$ were seeded at $30-40 \%$ confluence in 6 well plates. After $24 \mathrm{~h}$, cells reached $70-80 \%$ confluence and were then replaced with fresh medium. The transfection complex with human shRNA constructs was prepared immediately before adding to the cell growth medium as described previously (Liu et al. 2013). Briefly, 1 mg DNA was diluted in $250 \mu \mathrm{L}$ Opti-MEM medium (Gibco, Life Technology) and subjected to gentle vortexing. About $3 \mu \mathrm{L}$ TurboFectin 8.0 were added to the diluted DNA and pipetted gently to mix completely. The transfection complex was incubated for $15 \mathrm{~min}$ at room temperature; then the mixture was added to the cells in culture medium for transfection. The cells were incubated for $72 \mathrm{~h}$ and harvested; mRNA and protein were extracted separately. The efficiency of MFGE8 gene knockdown was determined by real-time PCR and immunoblotting.

\section{RNA extraction and quantitative real-time $P C R$}

Total RNA from transfected HEC-1A cells was isolated and quantified (RNeasy Mini Kit, Qiagen). Relative quantitative real-time PCR was performed using the LightCycler FastStart DNA MasterPlus SYBR Green I kit and the LightCycler 2.0 instrument (Roche Applied Science) to determine the MFGE8 mRNA. The PCR primers were designed based on published sequences of human MFGE8 from Invitrogen. The primers sequences were: forward GCCCTGGA TATCTGTTCCAA and reverse GCTCGACACATTTCGTCTCA; 18S, forward GTAACCCGTTGAACCCCATTC and reverse GCCGCACTAAACCATCCAATCG. mRNA expression was calculated as described previously (Yu et al. 2014). 
All results are presented as mean \pm S.E.M. and are based on at least three independent experiments.

\section{Protein extraction, SDS-PAGE and immunoblotting}

Total protein lysate was extracted using radioimmunoprecipitation assay lysis buffer supplemented with Halt Protease Inhibitor Cocktail (Pierce Thermo Scientific, Waltham, MA, USA). Protein was loaded and separated using $10 \%$ sodium dodecyl sulfate-polyacrylamide gel electrophoresis (SDS-PAGE) followed by electrotransfer onto a polyvinylidene fluoride membrane. Immunoblotting was performed using standard techniques as published previously (Yu et al. 2014).

For EMT analysis, E-cadherin and $\mathrm{N}$-cadherin protein expression was analyzed with anti-E-cadherin (1:2000; BD Biosciences) and anti-N-cadherin (1:2000; BD Biosciences) antibodies, and mesenchymal markers Snail (1:1000; Cell Signaling \#3895) and Vimentin (1:2500; BD Biosciences \#550513). For MFGE8 silencing work, MFGE8 protein expression from transfected HEC-1A cells was assessed using the anti-MFGE8 (1:1000; Abcam). Actin was used as loading control, anti-actin (1:1000; Abcam). Protein bands were detected by exposure to chemiluminescent substrate. Semiquantitative analysis was performed using Image J software (Yu et al. 2014). The semi-quantitative Western blotting was run in three independent experiments.

\section{Statistical analysis}

All results are presented as mean \pm S.E.M. and are based on at least three independent experiments. Statistical significances $(P$ value $\leq 0.05$.) were determined by applying one-way analysis of variance followed by Dunnett post hoc test among more than two groups and Student $t$-test between two groups, as appropriate.

\section{Results}

\section{TGF- $\beta$ induces morphological changes in endometrial epithelial cells}

HEC-1A cells underwent significant phenotypic changes after TGF- $\beta$ treatment. Images were captured by both light microscopy and AFM (Fig. 1). TGF- $\beta$ altered the size of the cells, rendering them wider, and changed the cobblestone-like epithelial morphology to spindle-, fibroblast-like shape. AFM probe was positioned over the cells in the red circle (Fig. 1A). For AFM, cells were scanned in contact mode. Based on deflection images, extensions around the cell (lamellipodia) were longer in TGF- $\beta$ treated group as compared with control. Additionally, TGF- $\beta$ altered cell height as measured from surface topographic AFM images. In the representative topography image (Fig. 1B), cell height in stimulated HEC-1A $(3.9 \mu \mathrm{m})$ was measured and was higher than that in control $(1.2 \mu \mathrm{m})$. Combined optical and AFM imaging of cells detected structural changes in individual cells including lamellipodia that were associated with cell spreading.
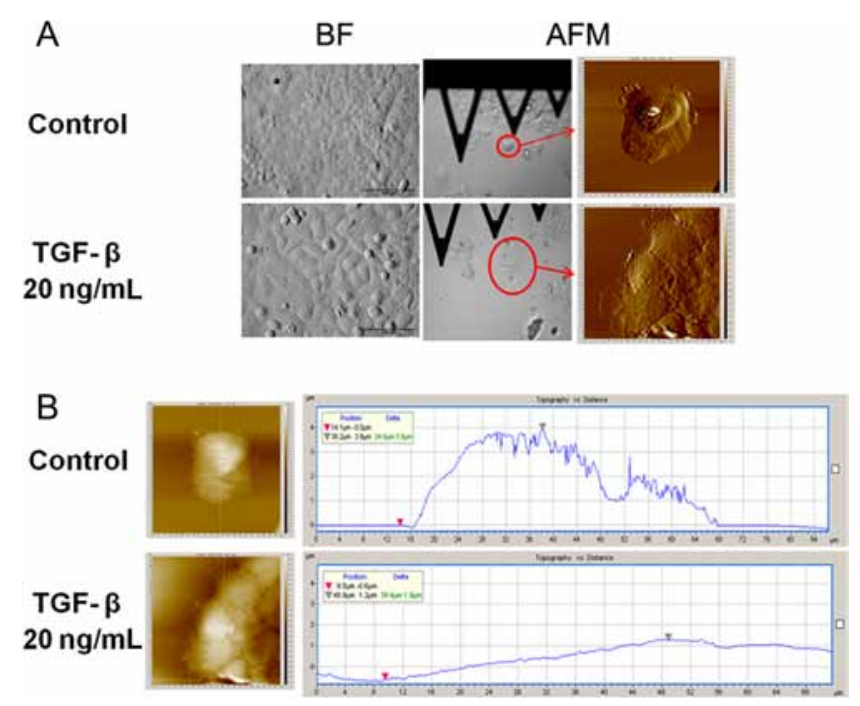

Figure 1 TGF- $\beta$-induced morphological changes in HEC-1A cells. Representative photographs of differential interference contrast and AFM images. (A) In bright field (BF), HEC-1A cells changed the cobblestone-like epithelial morphology to spindle-, fibroblast-like shape after TGF- $\beta$ treatment. In AFM image, cells were scanned in contact mode with probe positioning in red circle over the monolayer. Based on deflection images, extensions around the cell (lamellipodia) are longer in TGF- $\beta$-treated group as compared with controls. In the representative topography image (B), cell height in stimulated HEC-1A $(3.9 \mu \mathrm{m})$ was measured and was higher than that in control $(1.2 \mu \mathrm{m})$.

\section{TGF- $\beta$ induces EMT markers in endometrial epithelial cells to potentially stimulate early interactions of embryo and the endometrial epithelial cells}

TGF- $\beta$ ability to alter E-cadherin and $\mathrm{N}$-cadherin expressions was detected in HEC-1A cells. By immunofluorescence (Fig. 2A), the expression of E-cadherin was downregulated, whereas $\mathrm{N}$-cadherin expression was upregulated by TGF- $\beta$. These effects were also confirmed by changes in the protein levels of these molecular markers using immunoblotting (Fig. 2B). Immunoblotting results showed a remarkable decrease of E-cadherin and a significant increase of $\mathrm{N}$-cadherin after TGF- $\beta$ treatment $(P<0.05)$. We also found that other mesenchymal markers, such as Snail and Vimentin, were upregulated by TGF- $\beta$. Thus, TGF- $\beta$ induced EMT features accompanied by upregulation of the mesenchymal cell markers N-cadherin, Snail and Vimentin, with a concomitant downregulation of E-cadherin in HEC-1A cells. The semi-quantitative Western blotting was run in at least three independent experiments.

Embryo outgrowth was examined in an in vitro attachment/invasion assay. Mouse embryos (blastocysts) were placed on top of a plate with the monolayer HEC-1A cells with and without TGF- $\beta$ treatment, and monitored up to $72 \mathrm{~h}$ (Fig. 2C). All blastocysts in all the treatment groups retained a normal appearance during the co-culture. At the initiation of culture, no hatching 

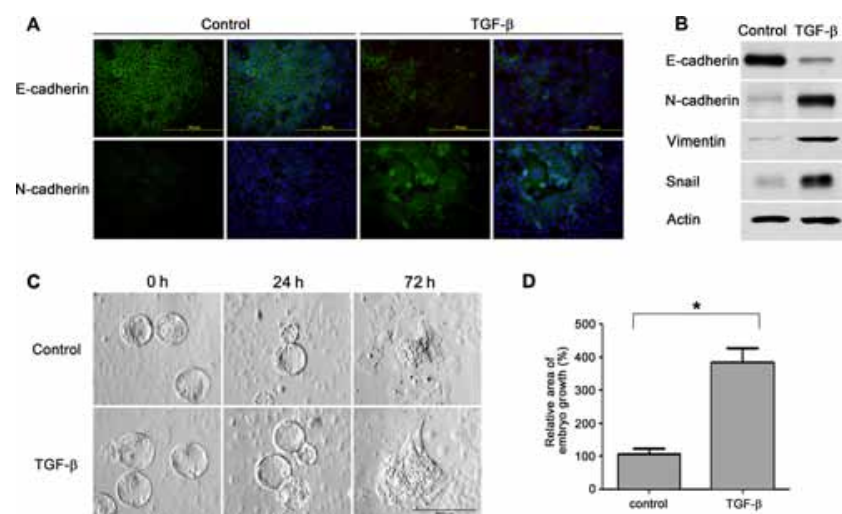

D

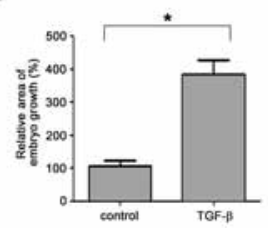

Figure 2 TGF- $\beta$-induced cadherin switch in HEC-1 A cells and altered mouse embryo outgrowth in the in vitro adhesion assay. Before the addition of mouse embryos, HEC-1A cell was pretreated with or without TGF- $\beta$. After 3-day treatment with TGF- $\beta$, a decrease of $\mathrm{E}$-cadherin expression and an increase of $\mathrm{N}$-cadherin levels were detected by immunofluorescence (A). This cadherin expression switch was also observed by Western blotting (B), which was run in three independent experiments. In a representative figure, E-cadherin and $\mathrm{N}$-cadherin were detected with their molecular weight at 120 and $130 \mathrm{kDa}$ respectively. We have also found the mesenchymal markers, such as Snail $(29 \mathrm{kDa})$ and Vimentin $(57 \mathrm{kDa})$, were upregulated by TGF- $\beta$. This cadherin expression switch and mesenchymal marker stimulation showed the ability of TGF- $\beta$ to promote EMT in HEC-1A cells. Mouse blastocysts were co-cultured with monolayer of HEC-1A, and evaluated up to $72 \mathrm{~h}$ (C). Embryo outgrowth area was measured and analyzed with Image J (D). Average outgrowth area of

blastocysts \pm S.E.M. cultured in different treatment groups were pooled from at least three experiments. A significant increase of outgrowth was detected from the TGF- $\beta$-treated HEC-1A cells as compared with control (embryo numbers $=18, P<0.05$ ).

was observed. After $24 \mathrm{~h}$, hatching, superficial spreading and outgrowth were observed, followed by adhesion/ attachment. After 72-h co-culture, the outgrowth area of mouse embryos from TGF- $\beta$ treated HEC-1A and control groups were compared (Fig. 2D). An increase of mouse embryo outgrowth area was detected in the TGF- $\beta$-treated group by Image J, and was shown as relative area of growth after normalization to control ( $n=18$ mouse embryos, $P<0.05$ vs controls). These results suggested that TGF- $\beta$-induced EMT is involved in trophoblast outgrowth.

\section{Knockdown of MFGE8 interferes with TGF- $\beta$ induced EMT}

To examine if MFG-E8 has an effect on the TGF- $\beta$ induced EMT, HEC-1A cells were transfected with shRNA-derived MFGE8 constructs. M1, M2, M3 and M4 are a set of shRNA constructs containing four vials of gene-specific shRNA expression vectors in retroviral silencing plasmid respectively. A negative control with non-effective shRNA cassette was used as a control (NC or nsRNA). MFGE8 mRNA (Fig. 3A) and protein expression (Fig. 3B) were examined. Compared with non-transfected cells, over $70 \%$ downregulation of
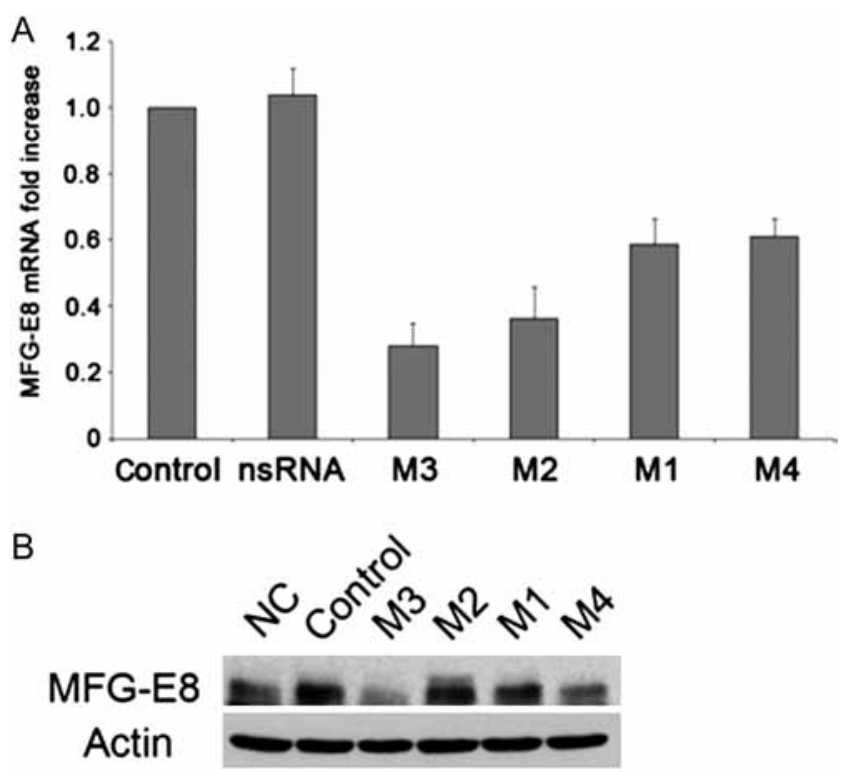

Figure 3 Knockdown of MFG-E8 by shRNA in HEC-1A cells. HEC-1A cells reached $70-80 \%$ confluence before transfection with shRNAderived MFG-E8 constructs. M1, M2, M3 and M4 are a set of shRNA constructs containing four vials of gene-specific shRNA expression vectors in retroviral silencing plasmid respectively. A negative control with non-effective shRNA cassette was used as a control (NC or nsRNA). After $72 \mathrm{~h}$, cells were collected for mRNA and protein level measurement. The quantitative real-time PCR and semi-quantitative Western blotting were presented as mean \pm S.E.M. and were based on at least three independent experiments. The efficiency of MFG-E8 gene knockdown was determined by real-time PCR (A) and Western blotting (B). HEC-1A transfected with M3 plasmid yield the most knockdown effects with $70 \%$ decrease in mRNA level and $90 \%$ decrease in protein expression as compared with the non-transfected cells $(P<0.05)$.

MFGE8 was achieved at the transcript level in cells transfected with M3 plasmids $(P<0.05)$. Western analysis confirmed the efficiency of transfection with M3 plasmid - that MFGE8 protein expression decreased $90 \%$ as compared with control $(P<0.05)$. A negative control transfected with non-effective shRNA cassette showed no significant difference as compared with nontransfected HEC-1A (NC, negative control).

To determine the effect of MFGE8 on TGF- $\beta$ induced EMT, HEC-1A cells were transfected with shRNA (M3 plasmids) and the E-cadherin expression was measured in mRNA level by real-time PCR (Fig. 4A), immunofluorescence (Fig. 4B), and protein level by Western blotting (Fig. 4C). In non-transfected HEC-1A cells, as expected, E-cadherin gene expression decreased $53 \%$ after TGF- $\beta$ treatment. This significant reduction was not observed in cells transfected with M3 plasmid $(P>0.05)$. Similarly, immunostaining results showed that TGF- $\beta$ altered E-cadherin distribution in treated cells as compared with controls. In confluent HEC-1A cells, E-cadherin expression was detected with distinctive organized localization at cell-cell borders. However, TGF- $\beta$ leads to redistribution 

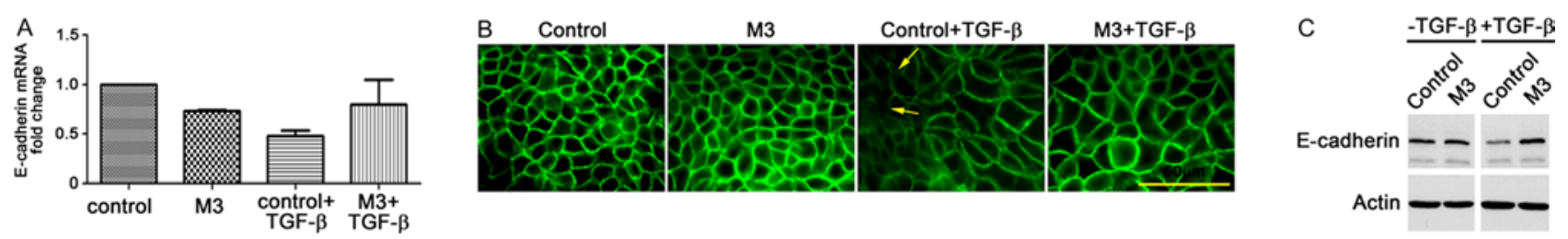

Figure 4 MFG-E8 silencing interfered with TGF- $\beta$-induced EMT. Transfected HEC-1A cells with shRNA MFG-E8 (M3 plasmids) were treated with TGF- $\beta$, and then E-cadherin expression was compared with control by measuring mRNA level using real-time PCR (A), protein level by immunofluorescence (B) and immunoblotting (C). In non-transfected HEC-1A cells, as expected, E-cadherin gene expression (A) decreased 53\% after TGF- $\beta$ treatment. This significant reduction was not observed in cells transfected with M3 plasmid $(P>0.05)$. Immunostaining (B) results detected E-cadherin redistribution when treated by TGF- $\beta$; this change was not found in M3-transfected HEC-1A cells. The arrows indicate the area that has reduced cell junction upon TGF- $\beta$ treatment. Similarly, the immunoblotting results (C) showed decreased E-cadherin expression by the induction of TGF- $\beta$; this reduction was not observed in the cells transfected with M3 plasmid. The quantitative real-time PCR and semiquantitative Western blotting were presented as mean \pm S.E.M. and were based on at least three independent experiments.

of adherent junctions with diffuse arrangement, although the fluorescent intensity was not found to be significantly different between groups. This change was not found in M3 transfected HEC-1A cells, suggesting that the knockdown of MFGE8 interfered with TGF- $\beta$ induced EMT. The immunoblotting results showed that E-cadherin expression in HEC-1A cells was decreased by the induction of TGF- $\beta$; this reduction was not observed in the cells transfected with M3 plasmid, which further confirmed the interference by MFGE8 of EMT. The quantitative real-time PCR and semi-quantitative Western blotting were presented as mean \pm S.E.M. and were based on three independent experiments.

\section{Discussion}

Human embryonic implantation is critical in establishing a pregnancy. A receptive endometrium is regulated across the menstrual cycle by ovarian steroids, which in addition to a plethora of local paracrine factors, establish the window of implantation (Gellersen \& Brosens 2014). The implantation process involves the processes of embryo apposition/adhesion to the luminal epithelial cells, followed by embryo penetration and invasion into the endometrial stromal cell layer, and later the blood vessels. Although several regulatory factors have been discovered as modulators of implantation, including both endometrial and embryonic origins (Jabbour et al. 2006, Cha et al. 2012), the intimate mechanism of how the endometrial barrier is disrupted and then remodeled is still largely unknown.

The EMT involves profound phenotypic changes that include the loss of cell-cell adhesion, the loss of cell polarity, and the acquisition of migratory and invasive properties ( $\mathrm{Li}$ et al. 2015). This process is frequently involved in various physiological and pathological events, such as embryonic development, cancer development, invasion and metastasis (Nieto 2013). It is known that unique cadherins are expressed during the initiation and progression of implantation (Paria et al. 1999), and that the change of some cadherins occurs along the entire length of the uterine epithelium in preparation for blastocyst adhesion (Hyland et al. 1998, Slater et al. 2002). We herein explored EMT of endometrial epithelial cells and their modifications in morphology and motility during the early stages of embryonic and endometrial epithelial cells interaction. MFGE8 has demonstrated functions in cell remodeling in breast cancer progression (Yang et al. 2011), and participates in immunomodulation and cell adhesion in the endometrium (Schmitz et al. 2014). In addition, findings in human melanoma cells revealed the promotion effects of MFGE8 on EMT in tumor microenvironment, where MFGE8 augmented cell resistance to apoptosis and stimulated invasion and immune suppression; they also found that MFGE8 knockdown attenuated Akt and Twist signaling and thereby compromised tumor cell survival and invasive ability (Jinushi et al. 2008). All these findings led us to hypothesize that MFGE8 plays an important role in regulating endometrial EMT during the endometrial epithelial barrier invasion and remodeling.

In this study, we used an in vitro model of human endometrial epithelial cells (HEC-1A) to examine EMT. As expected, the HEC- 1 A cells express ER- $\beta$, Prl-R, hCG-R, MFGE8, as well as epithelial markers cytokeratin 7 and 18, with negative expression of the mesenchymal protein vimentin (Supplementary Fig. 1). This follows our recent report that MFGE8 is expressed and is abundantly present in epithelial cells (luminal and glandular), where it promotes cell migration and apoptosis (Yu et al. 2014).

This study shows for the first time a putative role of MFGE8 protein in EMT of endometrial epithelial cells in vitro. To study the interactions at a cellular level, we first stimulated HEC-1A cells with a known EMT stimulator, i.e., TGF- $\beta$. The results of our experiments showed that HEC-1A cells undergo morphology/phenotypic changes in the presence of TGF- $\beta$, and concomitantly demonstrate a switch in cadherins expression, upregulation of other mesenchymal markers, such as Snail and Vimentin, strongly suggesting cell phenotypic and molecular changes of EMT. Decreased expression of E-cadherin on HEC-1A cells is the most consistent molecular marker for the epithelial disruption and remodeling as a result of a loss of cell to cell adhesion, increasing cell migration 
and cytoplasmic accumulation (Elston et al. 2009). EMT activation consists of several events that are associated with remodeling, such as epithelial disruption and degradation of the basement membrane, breakdown of epithelial tissue structure and ingression of the separated cells, and differentiation to the motile mesenchymal phenotype (Radisky \& Radisky 2010).

Bartley and coworkers demonstrated a co-expression of $\mathrm{E}$ - and $\mathrm{N}$-cadherin in endometriotic lesions and in eutopic normal proliferative endometrium at mRNA and protein levels (Bartley et al. 2014). They also found the first evidence for the expression of the transcription factors Twist, Snail and Slug in endometrium and in endometriosis lesions, known to downregulate E-cadherin expression in tumors during EMT. As stated by these authors, the co-expression of E- and $\mathrm{N}$-cadherin, along with the persistent expression of Twist in the endometrium, supports the idea that EMT is part of the highly dynamic cyclical changes in the endometrium, which enable it to proliferate each month after menstruation. Thus, the endometrium is one of the very few tissues where EMT seems to be part of a physiological process in adults.

Activated HEC-1A cells cause structural changes in individual cells after TGF- $\beta$ treatment. In this study, AFM captured morphology images of cells along with filopodia and lamellipodia that were associated with cell spreading and migration. During embryonic development, EMT takes place with construction of vertebral column out of the extracellular matrix that occurs in many physiology processes involved in cell migration. This mechanism of action also has been reported in the literature for EMT-mediated actions in other tissues. Migratory neural crest cells are involved with neuroectoderm as a result of EMT (Kalluri \& Weinberg 2009). These cells are dissociated from neural folds and gain motility. Mesenchymal morphology allows the cells to travel to specific targets and differentiate into many other cell types (Kerosuo \& Bronner-Fraser 2012). Additionally, Jha and coworkers have found that E-cadherin is expressed rather ubiquitously in the uterine epithelial cells. They observed the distinct enrichment on the apical membrane in the endometrium of periimplantation uterus specifically at the implantation sites and not at the inter-implantation sites (Jha et al. 2006). Thus, we propose that MFGE8 dependent signaling serves as a co-stimulus to TGF in the acquisition of mesenchymal properties via EMT that helps trophoblast attachment and early invasion.

In mammalian embryo development, a blastocyst is formed followed by a series of events in vivo, such as hatching from the zona pellucida, implantation and embryonic outgrowth (Lu et al. 2002). Here in this study, embryo outgrowth was defined according to the criteria that outgrowth distances reach half of the embryo's diameter with at least five giant trophoblast cells observed at the edge of outgrowing trophoblast cells
(Spindle \& Pedersen 1973). Our results demonstrated that cells undergo EMT and that this process could promote blastocyst growth, although hatching rate was not significantly affected by the co-culture of epithelial cells with TGF- $\beta$ pre-treatment (data not shown). This study used an in vitro model of attachment that uses mouse blastocysts and human endometrial epithelial cells. This involves a species crossover; although it is a well-documented model for attachment (as human embryo numbers are typically unavailable for research), the results should be interpreted cautiously (Uchida et al. 2012). Our observations suggest that MFGE8 and TGF- $\beta$ can cooperate in promoting embryo invasion potential. In addition, using shRNA to knockdown MFGE8 gene, we showed that TGF- $\beta$ induced EMT could be interfered in endometrial epithelial cells. These findings are supported by observations showing that MFGE8 is involved in tumor invasiveness via EMT in human melanoma cells (Jinushi et al. 2008).

In summary, TGF- $\beta$ induced EMT features in endometrial epithelial cells (HEC-1A), which were associated with the upregulation of the mesenchymal cell markers $\mathrm{N}$-cadherin, Snail and Vimentin, with a concomitant downregulation of E-cadherin. We also demonstrated, for the first time, that MFGE8 plays a modulatory role in endometrial epithelial cell EMT, an important process taking place at the implantation site. We have tested and confirmed the ability of TGF- $\beta$ to induce EMT in endometrial epithelial cells in vitro, and by using MFGE8 shRNA, we show that this EMT process is interfered, with downregulation of EMT biomarkers when MFGE8 is silenced.

Endometrial epithelial cell migration through EMT plays an important role in the remodeling of the luminal epithelial barrier during implantation (Uchida et al. 2012). These studies reveal a potential physiological role of MFGE8 in facilitating TGF-induced EMT, which in turn would favor trophoblast attachment, outgrowth (with early invasion) and epithelial remodeling at the implantation site. Our investigations reveal a mechanism by which endometrial epithelial cells may capitalize on autocrine or paracrine MFGE8 secretion to maintain the cross talk between embryo and endometrium. MFGE8 blockage might also antagonize embryo invasion and thereby increase miscarriage.

\section{Supplementary data}

This is linked to the online version of the paper at http://dx.doi.org/10.1530/REP-15-0585.

\section{Declaration of interest}

The authors declare that there is no conflict of interest that could be perceived as prejudicing the impartiality of the research reported. 


\section{Funding}

This work was supported by the Jones Institute Foundation and EVMS Research Grant 572822 to Silvina Bocca, MD, and the National Natural Science Foundation of China Grant 31501209 to Liang Yu, PhD.

\section{References}

Bartley J, Julicher A, Hotz B, Mechsner S \& Hotz H 2014 Epithelial to mesenchymal transition (EMT) seems to be regulated differently in endometriosis and the endometrium. Archives of Gynecology and Obstetrics 289 871-881. (doi:10.1007/s00404-013-3040-4)

Bertrand M, Petit V, Jain A, Amsellem R, Johansen T, Larue L, Codogno P \& Beau I 2015 SQSTM1/p62 regulates the expression of junctional proteins through epithelial-mesenchymal transition factors. Cell Cycle 14 364-374. (doi:10.4161/15384101.2014.987619)

Bocca SM, Anderson S, Amaker B, Swanson RJ, Franchi A, Lattanzio F \& Oehninger S 2012 Milk fat globule epidermal growth factor 8 (MFG-E8): a novel protein in the mammalian endometrium with putative roles in implantation and placentation. Placenta 33 795-802. (doi:10.1016/j. placenta.2012.06.015)

Burch T, Yu L, Nyalwidhe J, Horcajadas JA, Bocca S, Swanson RJ \& Oehninger S 2014 Characterization of secreted proteins of 2-cell mouse embryos cultured in vitro to the blastocyst stage with and without protein supplementation. Journal of Assisted Reproduction and Genetics 31 757-765. (doi:10.1007/s10815-014-0207-2)

Cha J, Sun X \& Dey SK 2012 Mechanisms of implantation: strategies for successful pregnancy. Nature Medicine 18 1754-1767. (doi:10.1038/ nm.3012)

Chea HK, Wright CV \& Swalla BJ 2005 Nodal signaling and the evolution of deuterostome gastrulation. Developmental Dynamics 234 269-278. (doi:10.1002/(ISSN)1097-0177)

Cheng JC, Auersperg N \& Leung PC 2012 TGF-beta induces serous borderline ovarian tumor cell invasion by activating EMT but triggers apoptosis in low-grade serous ovarian carcinoma cells. PLoS One 7 e42436. (doi:10.1371/journal.pone.0042436)

Elston MS, Gill AJ, Conaglen JV, Clarkson A, Cook RJ, Little NS, Robinson BG, Clifton-Bligh RJ \& McDonald KL 2009 Nuclear accumulation of E-cadherin correlates with loss of cytoplasmic membrane staining and invasion in pituitary adenomas. Journal of Clinical Endocrinology and Metabolism 94 1436-1442. (doi:10.1210/ jc.2008-2075)

Ensslin MA \& Shur BD 2003 Identification of mouse sperm SED1, a bimotif EGF repeat and discoidin-domain protein involved in sperm-egg binding. Cell 114 405-417. (doi:10.1016/S0092-8674(03)00643-3)

Franchi A, Bocca S, Anderson S, Riggs R \& Oehninger S 2011 Expression of milk fat globule EGF-factor 8 (MFG-E8) mRNA and protein in the human endometrium and its regulation by prolactin. Molecular Human Reproduction 17 360-371. (doi:10.1093/molehr/gaq102)

Gellersen B \& Brosens J 2014 Cyclic decidualization of the human endometrium in reproductive health and failure. Endocrine Reviews 35 851-905. (doi:10.1210/er.2014-1045)

Green CJ, Fraser ST \& Day ML 2015 Insulin-like growth factor 1 increases apical fibronectin in blastocysts to increase blastocyst attachment to endometrial epithelial cells in vitro. Human Reproduction 30 284-298. (doi:10.1093/humrep/deu309)

Hyland RA, Shaw TJ, Png FY \& Murphy CR 1998 Pan-cadherin concentrates apically in uterine epithelial cells during uterine closure in the rat. Acta Histochemica 100 75-81. (doi:10.1016/S0065-1281(98)80007-X)

Jabbour HN, Kelly RW, Fraser HM \& Critchley HO 2006 Endocrine regulation of menstruation. Endocrine Reviews 27 17-46. (doi:10.1210/ er.2004-0021)

Jha RK, Titus S, Saxena D, Kumar PG \& Laloraya M 2006 Profiling of E-cadherin, beta-catenin and $\mathrm{Ca}(2+)$ in embryo-uterine interactions at implantation. FEBS Letters $\mathbf{5 8 0}$ 5653-5660. (doi:10.1016/j. febslet.2006.09.014)

Jinushi M, Nakazaki Y, Carrasco DR, Draganov D, Souders N, Johnson M, Mihm MC \& Dranoff G 2008 Milk fat globule EGF-8 promotes melanoma progression through coordinated Akt and twist signaling in the tumor microenvironment. Cancer Research 68 8889-8898. (doi:10.1158/0008-5472.CAN-08-2147)

Jones RL, Stoikos C, Findlay JK \& Salamonsen LA 2006 TGF-beta superfamily expression and actions in the endometrium and placenta. Reproduction 132 217-232. (doi:10.1530/rep.1.01076)

Kalluri R \& Weinberg RA 2009 The basics of epithelial-mesenchymal transition. Journal of Clinical Investigation 119 1420-1428. (doi:10.1172/ JCI39104)

Kawamura K, Chen Y, Shu YM, Cheng Y, Qiao J, Behr B, Pera RAR \& Hsueh AJW 2012 Promotion of human early embryonic development and blastocyst outgrowth in vitro using autocrine/paracrine growth factors. PLOS ONE 7 e49328. (doi:10.1371/journal.pone.0049328)

Kerosuo L \& Bronner-Fraser M 2012 What is bad in cancer is good in the embryo: Importance of EMT in neural crest development. Seminars in Cell and Developmental Biology 23 320-332. (doi:10.1016/j. semcdb.2012.03.010)

Li Z, Gou J, Jia J \& Zhao X 2015 MicroRNA-429 functions as a regulator of epithelial-mesenchymal transition by targeting Pcdh8 during murine embryo implantation. Human Reproduction 30 507-518. (doi:10.1093/ humrep/dev001)

Liu Y, Yang X, Guo C, Nie P, Liu Y \& Ma J 2013 Essential role of MFG-E8 for phagocytic properties of microglial cells. PLoS One 8 e55754. (doi:10.1371/journal.pone.0055754)

Lu DP, Tian L, O'Neill C \& King NJC 2002 Regulation of cellular adhesion molecule expression in murine oocytes, peri-implantation and postimplantation embryos. Cell Research 12 373-383. (doi:10.1038/ sj.cr.7290139)

Massague J, Blain SW \& Lo RS 2000 TGF beta signaling in growth control, cancer, and heritable disorders. Cell 103 295-309. (doi:10.1016/S00928674(00)00121-5)

Mirkin S, Arslan M, Churikov D, Corica A, Diaz J, Williams S, Bocca S \& Oehninger S 2005 In search of candidate genes critically expressed in the human endometrium during the window of implantation. Human Reproduction 20 2104-2117. (doi:10.1093/ humrep/dei051)

Morris HB, Gatter KC, Pulford K, Haynes P, Charnock M, TaylorPapadimitriou J, Lane EB \& Mason DY 1983 Cervical wart virus infection, intraepithelial neoplasia and carcinoma; an immunohistological study using a panel of monoclonal antibodies. British Journal of Obstetrics and Gynaecology 90 1069-1081. (doi:10.1111/bjo.1983.90.issue-11)

Nieto MA 2013 Epithelial plasticity: a common theme in embryonic and cancer cells. Science 342 708. (doi:10.1126/science.1234850)

Omwandho CO, Konrad L, Halis G, Oehmke F \& Tinneberg HR 2010 Role of TGF-betas in normal human endometrium and endometriosis. Human Reproduction 25 101-109. (doi:10.1093/humrep/dep382)

Paria BC, Zhao X, Das SK, Dey SK \& Yoshinaga K 1999 Zonula occludens-1 and E-cadherin are coordinately expressed in the mouse uterus with the initiation of implantation and decidualization. Developmental Biology 208 488-501. (doi:10.1006/dbio.1999.9206)

Paule S, Aljofan M, Simon C, Rombauts LJF \& Nie GY 2012 Cleavage of endometrial -integrins into their functional forms is mediated by proprotein convertase 5/6. Human Reproduction 27 2766-2774. (doi:10.1093/humrep/des203)

Piestrzeniewicz-Ulanska D, Brys $M$, Semczuk A, Jakowicki JA \& Krajewska WM 2002 Expression of TGF-beta type I and II receptors in normal and cancerous human endometrium. Cancer Letters 186 231-239. (doi:10.1016/S0304-3835(02)00351-8)

Qin J, Takahashi Y, Isuzugawa K, Imai M, Yamamoto S, Hirai Y \& Imakawa K 2005 Regulation of embryo outgrowth by a morphogenic factor, epimorphin, in the mouse. Molecular Reproduction and Development 70 455-463. (doi:10.1002/(ISSN)1098-2795)

Radisky DC, Levy DD, Littlepage LE, Liu H, Nelson CM, Fata JE, Leake D, Godden EL, Albertson DG, Nieto MA et al. 2005 Rac1b and reactive oxygen species mediate MMP-3-induced EMT and genomic instability. Nature 436 123-127. (doi:10.1038/nature03688)

Radisky ES \& Radisky DC 2010 Matrix metalloproteinase-induced epithelial-mesenchymal transition in breast cancer. Journal of Mammary Gland Biology and Neoplasia 15 201-212. (doi:10.1007/s10911-0109177-x)

Rhyu DY, Yang Y, Ha H, Lee GT, Song JS, Uh ST \& Lee HB 2005 Role of reactive oxygen species in TGF-beta1-induced mitogen-activated protein kinase activation and epithelial-mesenchymal transition in renal 
tubular epithelial cells. Journal of the American Society of Nephrology 16 667-675. (doi:10.1681/ASN.2004050425)

Sarhan A, Bocca S, Yu L, Anderson S, Jacot T, Burch T, Nyalwidhe J, Sullivan C, Kaur M, Bajic VB et al. 2013 Human endometrial milk fat globule-epidermal growth factor 8 (MFGE8) is up regulated by estradiol at the transcriptional level, and its secretion via microvesicles is stimulated by human chorionic gonadotropin (hCG). Cell Signalling and Trafficking 1 1. (doi:10.7243/2054-1481-1-1)

Schmitz C, Yu L, Bocca S, Anderson S, Cunha-Filho JS, Rhavi BS \& Oehninger S 2014 Role for the endometrial epithelial protein MFG-E8 and its receptor integrin alphavbeta3 in human implantation: results of an in vitro trophoblast attachment study using established human cell lines. Fertility and Sterility 101 874-882. (doi:10.1016/j. fertnstert.2013.12.015)

Silvestre JS, Thery C, Hamard G, Boddaert J, Aguilar B, Delcayre A, Houbron C, Tamarat R, Blanc-Brude O, Heeneman S et al. 2005 Lactadherin promotes VEGF-dependent neovascularization. Nature Medicine 11 499-506. (doi:10.1038/nm1233)

Slater M, Murphy CR \& Barden JA 2002 Tenascin, E-cadherin and P2X calcium channel receptor expression is increased during rat blastocyst implantation. Histochemical Journal 34 13-19. (doi:10.1023/ A:1021335606896)

Spindle Al \& Pedersen RA 1973 Hatching, attachment, and outgrowth of mouse blastocysts in vitro: fixed nitrogen requirements. Journal of Experimental Zoology 186 305-318. (doi:10.1002/(ISSN)1097-010X)

Stubbs JD, Lekutis C, Singer KL, Bui A, Yuzuki D, Srinivasan U \& Parry G 1990 cDNA cloning of a mouse mammary epithelial cell surface protein reveals the existence of epidermal growth factor-like domains linked to factor VIII-like sequences. PNAS 87 8417-8421. (doi:10.1073/ pnas.87.21.8417)

Taylor MR, Couto JR, Scallan CD, Ceriani RL \& Peterson JA 1997 Lactadherin (formerly BA46), a membrane-associated glycoprotein expressed in human milk and breast carcinomas, promotes Arg-Gly-Asp (RGD)-dependent cell adhesion. DNA and Cell Biology 16 861-869. (doi:10.1089/dna.1997.16.861)

Thiery JP, Acloque H, Huang RY \& Nieto MA 2009 Epithelial-mesenchymal transitions in development and disease. Cell 139 871-890. (doi:10.1016/j.cell.2009.11.007)
Tsukamoto H, Shibata K, Kajiyama H, Terauchi M, Nawa A \& Kikkawa F 2007 Irradiation-induced epithelial-mesenchymal transition (EMT) related to invasive potential in endometrial carcinoma cells. Gynecologic Oncology 107 500-504. (doi:10.1016/j.ygyno.2007.08.058)

Uchida H, Maruyama T, Nishikawa-Uchida S, Oda H, Miyazaki K, Yamasaki A \& Yoshimura Y 2012 Studies using an in vitro model show evidence of involvement of epithelial-mesenchymal transition of human endometrial epithelial cells in human embryo implantation. Journal of Biological Chemistry 287 4441-4450. (doi:10.1074/jbc. M111.286138)

Van Themsche C, Mathieu I, Parent S \& Asselin E 2007 Transforming growth factor-beta3 increases the invasiveness of endometrial carcinoma cells through phosphatidylinositol 3-kinase-dependent up-regulation of X-linked inhibitor of apoptosis and protein kinase c-dependent induction of matrix metalloproteinase-9. Journal of Biological Chemistry 282 4794-4802. (doi:10.1074/jbc.M608497200)

Yang CW, Hayashida T, Forster N, Li CQ, Shen DJ, Maheswaran S, Chen L, Anderson KS, Ellisen LW, Sgroi D et al. 2011 The integrin alpha(v)beta(35) ligand MFG-E8 is a p63/p73 target gene in triple-negative breast cancers but exhibits suppressive functions in $\mathrm{ER}+$ and erbB2(+) breast cancers. Cancer Research 71 937-945. (doi:10.1158/0008-5472.CAN-10-1471)

Yang J \& Weinberg RA 2008 Epithelial-mesenchymal transition: at the crossroads of development and tumor metastasis. Developmental Cell 14 818-829. (doi:10.1016/j.devcel.2008.05.009)

Yu L, Anderson S, Oehninger S \& Bocca S 2014 Tumor necrosis factor alpha up-regulates endometrial milk fat globule-epidermal growth factor 8 protein production via nuclear factor kappaB activation, resulting in cell migration of epithelial cells. Fertility and Sterility 101 552-559. (doi:10.1016/j.fertnstert.2013.10.032)

Received 14 December 2015

First decision 11 January 2016

Revised manuscript received 2 May 2016

Accepted 17 June 2016 